\title{
Star Formation in Tadpole Galaxies
}

\author{
Casiana Muñoz-Tuñón ${ }^{1,2}$, Jorge Sánchez Almeida ${ }^{1,2}$, Debra M. Elmegreen ${ }^{3}$, \\ Bruce G. Elmegreen ${ }^{4}$ \\ ${ }^{1}$ Instituto de Astrofísica de Canarias, E-38205 La Laguna, Tenerife, Spain \\ ${ }^{2}$ Departamento de Astrofísica, Universidad de La Laguna, Tenerife, Spain \\ ${ }^{3}$ Department of Physics and Astronomy, Vassar College, Poughkeepsie, NY 12604, USA \\ ${ }^{4}$ IBM Research Division, T.J. Watson Research Center, Yorktown Heights, NY 10598, USA \\ Corresponding author: cmt@iac.es
}

\begin{abstract}
Tadpole Galaxies look like a star forming head with a tail structure to the side. They are also named cometaries. In a series of recent works we have discovered a number of issues that lead us to consider them extremely interesting targets. First, from images, they are disks with a lopsided starburst. This result is firmly established with long slit spectroscopy in a nearby representative sample. They rotate with the head following the rotation pattern but displaced from the rotation center. Moreover, in a search for extremely metal poor (XMP) galaxies, we identified tadpoles as the dominant shapes in the sample - nearly $80 \%$ of the local XMP galaxies have a tadpole morphology. In addition, the spatially resolved analysis of the metallicity shows the remarkable result that there is a metallicity drop right at the position of the head. This is contrary to what intuition would say and difficult to explain if star formation has happened from gas processed in the disk. The result could however be understood if the star formation is driven by pristine gas falling into the galaxy disk. If confirmed, we could be unveiling, for the first time, cool flows in action in our nearby world. The tadpole class is relatively frequent at high redshift $-10 \%$ of resolvable galaxies in the Hubble UDF but less than $1 \%$ in the local Universe. They are systems that could track cool flows and test models of galaxy formation.
\end{abstract}

Keywords: starburst galaxies - tadpoles - cool flows - rotation curves - galaxy disks.

\section{Introduction}

Elongated galaxies with bright clumps at one end are visible in deep field images taken with HST or from the ground; van der Bergh et al., 1996 called them "tadpole" galaxies". Figure 1 in Elmegreen et al. (2005) shows different morphologies of galaxies observed with the Hubble Ultra Deep Field (UDF). The fourth row presents images of Tadpoles. This asymmetric morphology is rather common at high redshift but rare in the local universe. For example, tadpoles constitute $10 \%$ of all galaxies larger than 10 pixels in the UDF (Elmegreen et al. 2007; Elmegreen \& Elmegreen, 2010), and they represent $6 \%$ of the UDF galaxies identified by Straughn et al. (2006) and Windshorst et al. (2006) using automated search algorithms.

In contrast, Elmegreen et al. (2012; hereafter Paper I), find only $0.2 \%$ tadpoles among the uv-bright local galaxies of the Kiso survey by Miyauchi-Isobe et al. (2010). This decrease suggests that the tadpole morphology represents a common but transition phase during the assembly of some galaxies. Since local tadpole galaxies are very low mass objects compared to their high redshift analogues, this phase must be already over for the local descendants of high redshift tadpoles.

The tadpole structure has inspired several explanations, such as ram pressure stripping that triggers star formation at the leading edge, to mergers (see Sánchez Almeida et al. 2013 for an extensive review). The explanation that we propose, based on observational evidence summarized here, is that the starburst head may result from the accretion of an external flow of pristine gas that penetrates the dark matter halo and hits and heats a pre-existing disk, which is viewed to the side as the tail.

We will briefly present a summary of recent results showing, first that the local Tadpoles share the properties of their higher redshift and higher mass counterparts (section 2), that they belong to the extremely metal poor (XMP) sample of the Blue Compact Dwarfs family (section 3), they are rotating discs (section 4) and, finally, that the starbursts (heads) show a drop in the already low metallicity that can only be understood if fresh metal-poor gas is falling onto the galaxy (section 
5). We finish with a brief summary and future actions.

\section{Local and Hight z TPG Share Properties}

We used Sloan Digital Sky Survey data to determine the ages, masses, and surface densities of the heads and tails in 14 local tadpoles (shown in Figure 1) selected from the Kiso and Michigan surveys of UV-bright galaxies, and we compared them to Tadpoles previously studied in the Hubble Ultra Deep Field. The result, published in Paper I is that the young stellar mass in the head scales linearly with the rest-frame galaxy luminosity, ranging from $\approx 10^{5} M_{\odot}$ at galaxy absolute magnitude $U=-13 \mathrm{mag}$ to $10^{9} M_{\odot}$ at $U=-20 \mathrm{mag}$. The tails in the local sample look like bulge-free galaxy disks. Their photometric ages decrease from several Gyr to several hundred Myr with increasing redshift. The far-outer intensity profiles in the local sample are symmetric and exponential. We suggest that most local tadpoles are bulge-free galaxy disks with lopsided star formation, perhaps from environmental effects such as ram pressure or disk impacts, or from in-situ gas collapse to a giant star-forming clump with a Jeans-length that is comparable to half the disk size. The existence of a disk, proposed from the analysis of the luminosity profiles, has been further confirmed spectroscopically (see section 4).

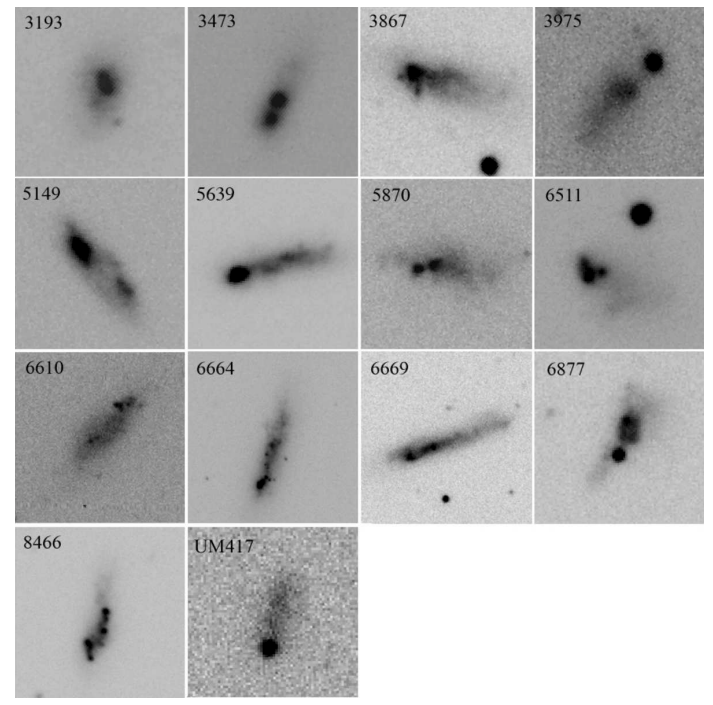

Figure 1: Tadpole galaxies from the Kiso and UM samples. Figure from Elmegreen et al., 2012.

\section{Belong to the BCDs- XMP Class}

Blue Compact Galaxies are important targets for a number of reasons. They are small systems, thought to remain as fossils or debris from the formation of larger galaxies in the early epochs of the Universe. Their low metallicities, high sSFRs and low doubling time (less than 1Gy) and active stabursts make them ideal targets to study the old Universe at low redshift. Their complete census, as well as the likelihood that they have a long quiescent phase between starbursts, took us to study the whole sample by making use of SDSS. The properties of the quiescent and burst phases were determined from detailed studies of the bursts and host galaxies of a nearby sample (Amorin et. al., 2007, 2008). These properties allowed us to identify such objects in the database. The SDSS DR6 database provides $\approx 21.500$ quiescent $\mathrm{BCD}$ candidates, a number 30 times larger to those bursting (BCDs). This result implies that one out of every three dwarf galaxies in the local universe may be a quiescent BCD. The properties of the two samples are consistent with a single sequence in galactic evolution with the quiescent phase lasting 30 times longer than the burst phase (Sánchez Almeida et. al., 2008). In Sánchez Almeida et al. (their figure 9) there is a clear subsample of objects with the lowest luminosity which turn out to be also those that are most metal poor.

We carried out a systematic search for extremely metal-poor (XMP) galaxies in the spectroscopic sample of Sloan Digital Sky Survey (SDSS) data release 7 (DR7) (Morales-Luis et al., 2011). The XMP candidates are found by classifying all of the galaxies according to the form of their spectra in a region $80 \AA$ wide around $\mathrm{H} \alpha$ using an automatic classification algorithm, k-means (Sánchez Almeida et al.,2009). Our systematic search renders 32 galaxies having negligible [N II] lines, as expected in XMP galaxy spectra. Twenty-one of them were previously identified as XMP galaxies in the literature, and 11 were new. This was established after a thorough bibliographic search that yielded only some 130 galaxies known to have an oxygen metallicity 10 times smaller than the Sun (explicitly, with $12+\log$ $[\mathrm{O} / \mathrm{H}] \leq 7.65)$. XMP galaxies are rare; they represent $0.01 \%$ of the emission lines galaxies in SDSS/DR7. The XMP galaxies constitute $0.1 \%$ of the galaxies in the local volume, or $\approx 0.2 \%$ of the emission-line galaxies. All but four of our candidates are BCD galaxies, and 24 of them have either cometary shape or are formed by chained knots. Note that this result, the morphology, is absolutely independent of our search criterium (spectra).

\section{Tadpoles are Rotating Disks}

The work in Paper I was followed up by mean of high spectral resolution long slit observations at the INT $(2.5 \mathrm{~m})$ at Observatorio del Roque de los Muchachos (ORM) with IDS spectrograph. In order to determine the dynamical properties and metallicities of lo- 

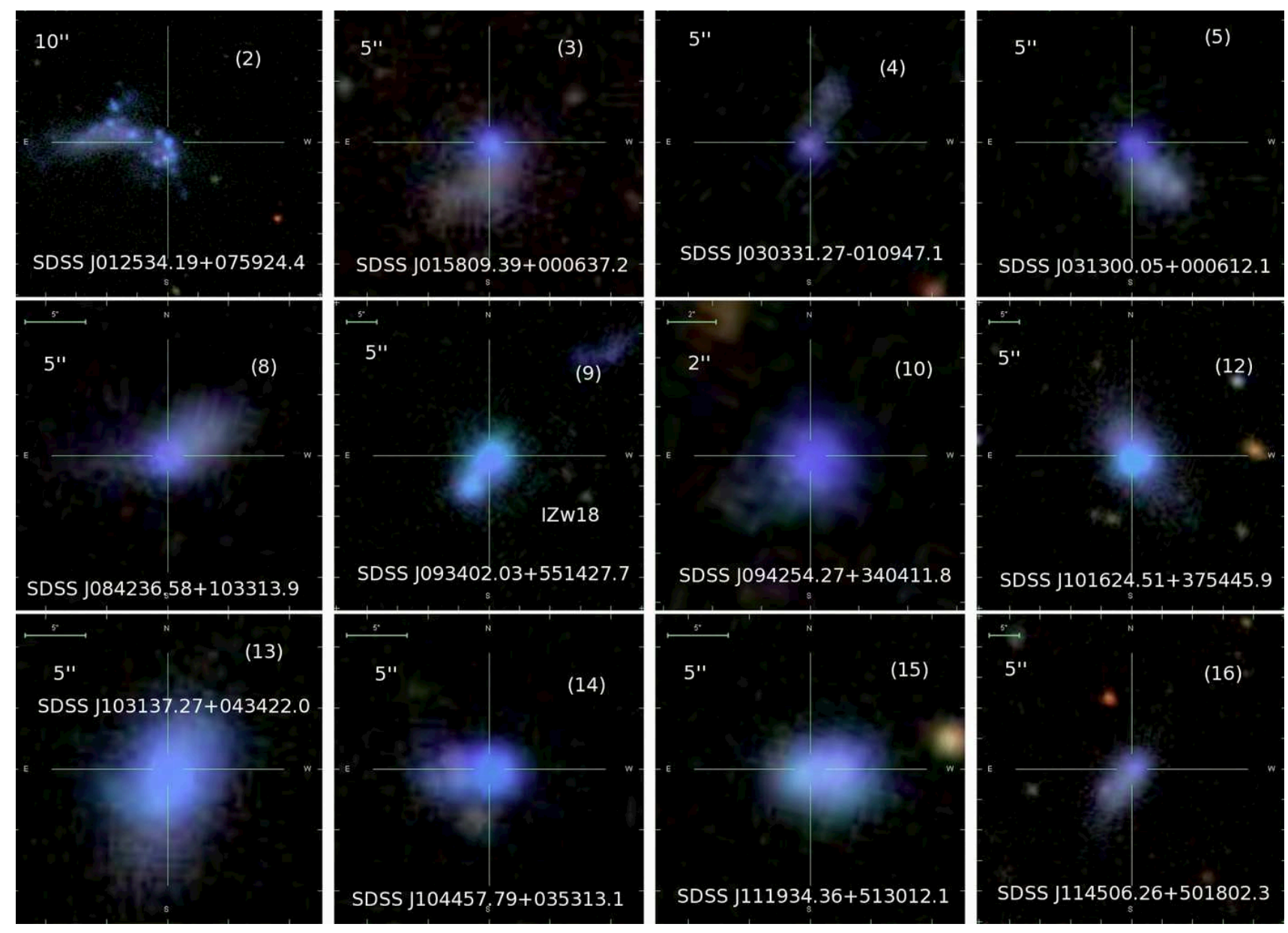

Figure 2: SDSS mugshots of all XMP candidates (from spectroscopy). The figure is taken from Morales-Luis et al., 2011. Note the prevalence of Tadpole shapes.

cal tadpoles, we measured $\mathrm{H} \alpha$ spectra along the headtail direction in a representative fraction of the original sample. Further details are in Sánchez Almeida et al. (2013). In Figure 3, we show spectral flux (the solid lines) and $\mathrm{H} \alpha$ flux (the dashed lines) along the slit. Note the obvious lopsidedness of the light distributions, as expected from the tadpole shape. The origin of distance on the abscissa has been set as the position of the maximum of the spectral flux distribution. The dotted line represents a Gaussian fitted to the $\mathrm{H} \alpha$ flux around the head. The horizontal bar in each panel gives a common length scale corresponding to $1 \mathrm{kpc}$. The thin vertical solid lines indicate the center of rotation obtained from the rotation curve fit shown in Fig. 4.

Velocities, masses, abundances and other physical parameters were determined from the spectra. Bulk velocities were measured from the displacement of $\mathrm{H} \alpha$. We computed the displacement both as the barycenter of the emission line, and as the center of a Gaus- sian function fitted to the profile. Errors were estimated from the $\mathrm{S} / \mathrm{N}$ measured in the continuum and then propagated to the centroids. The FWHM of the profiles were also measured directly from the profiles and from the Gaussian fits. Their errors were also inferred from the noise measured in the continuum by error propagation.

Figure 4 shows the velocity curves of the tadpole galaxies included in the study. The abscissae represent distances along the major axes of the galaxies from the position of the tadpole head; i.e., the brightest point on the galaxy. The range of distances differs for the different targets, but the horizontal bar in each panel gives a common length scale corresponding to $1 \mathrm{kpc}$. The points with error bars show the observations whereas the thick solid line represents the best fit of the observed points to the analytic rotation curve. The part of the rotation curve shown in red indicates the portion of the velocity curve used for fitting. The thin horizon- 
tal and vertical lines indicate the systemic velocity and the center of rotation obtained from the fit, respectively. The little arrows on top of each panel also indicate the centers of rotation. The zero of the velocity scale is set by velocity of the spatially integrated spectrum of the galaxy; positive velocities are redshifts.
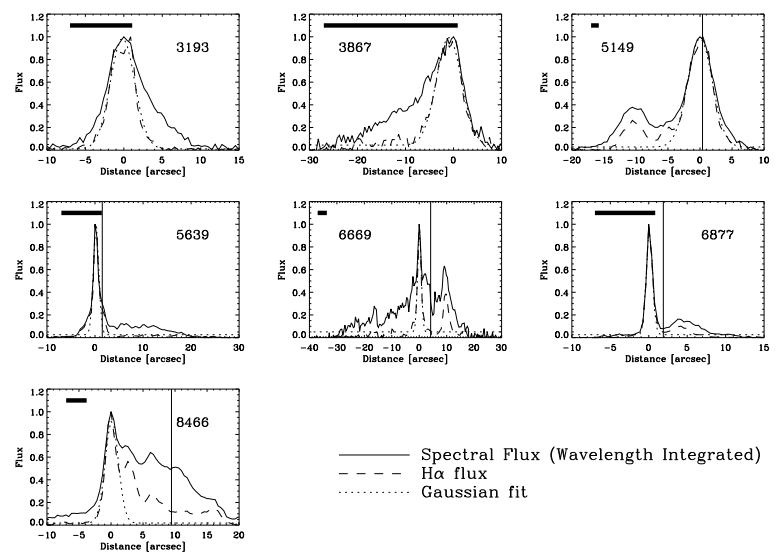

Figure 3: Spectral flux (the solid lines) and $\mathrm{H} \alpha$ flux (the dashed lines). Formal error bars for photometry are not included since they are negligibly small.

The main result shown in Figure 4 is that five out of seven targets show velocity gradients interpreted as rotation. Another important result is that the tadpole head is not at the rotation center.
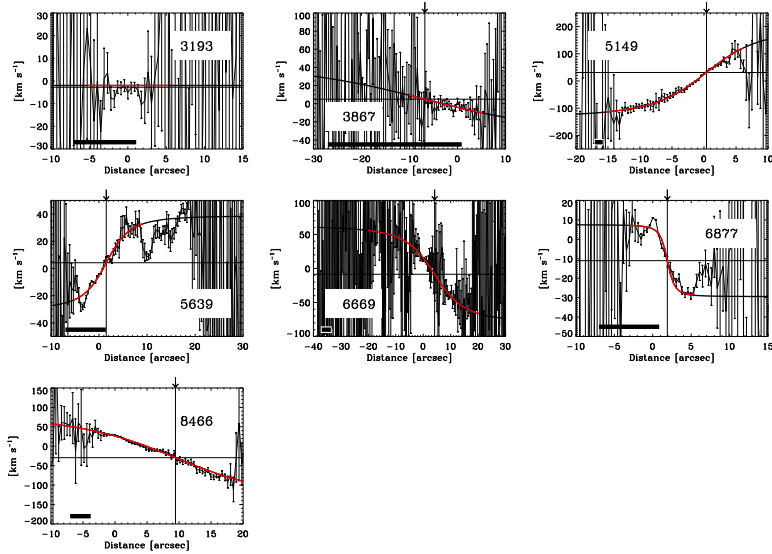

Figure 4: Velocity curves of the seven tadpole galaxies obtained from long slit spectroscopy. Figure from Sánchez Almeida et al. 2013

Sometimes the interpretation of the velocity curve as rotation is obvious (e.g., KISO8466), but other times the curve looks more like a perturbed rotation (e.g., KISO5639). KISO3193 and KISO3867 have a rather flat velocity curve, and therefore no obvious rotation. However, one of them, KISO3867, shows a systematic line shift of the order $10-20 \mathrm{~km} \mathrm{~s}^{-1}$ between the two ex- tremes of the galaxy (Figure 4). The amplitude is of the order of the error bars, but the displacement is in the raw data as judged by inspection of the individual $\mathrm{H} \alpha$ profiles.

\section{Drops in Metallicity at the Head Location}

The metallicity along the slit was estimated using the ratio $[\mathrm{NII}] \lambda 6583$ to $\mathrm{H} \alpha$. Figure 5 taken from Sánchez Almeida et al (2013) shows the variation across the galaxies of the oxygen abundance, including their error bars. All galaxies have sub-solar metallicity - the thick horizontal line marks the solar oxygen abundance given by $12+\log (\mathrm{O} / \mathrm{H})_{\odot}=8.69 \pm 0.05$ (Asplund et al., 2009). The galaxies also present significant abundance gradients, with the lowest abundances tending to coincide with the largest $\mathrm{H} \alpha$ emissions (e.g., KISO6669 and KISO6877) in Fig. 5. Remember that the vertical dotted lines mark the position of the peak $\mathrm{H} \alpha$ fluxes. Two targets, KISO5639 and KISO6877, have metallicities well below one-tenth the solar value, therefore, they belong to the XMP galaxies class (Kunth \& Östlin, 2000).

XMP galaxies are really rare objects: one out of a thousand galaxies in the local universe according to Morales-Luis et al. (2011). Therefore the fact that we observe two in a sample of seven cannot be a coincidence. It is known that a significant fraction of XMP galaxies turn out to be cometary or tadpole (Papaderos et al., 2008). We have found that the reverse holds too, i.e., that tadpole galaxies have a significant probability of being XMP. This fact supports the idea that the tadpole morphology is a sign of dynamical youth, as the low metallicity is a sign of being chemically young (see discussion in Sánchez Almeida et al., 2013).

\section{Summary and Future}

Tadpole Galaxies have an easily identified shape, belong to the Blue Compact Dwarf class, and are rare in the nearby Universe. Their appearance suggests then to be the consequence of some interaction or star formation triggered by ram pressure processes. These local tadpoles seem though to form a continuous sequence with the UDF Tadpoles, seen in relatively larger number at high redshift (Elmegreen \& Elmegreen 2010). Regarding their photometric properties, local Tadpoles occupy the low mass end in sequences such as star formation, surface density and mass-to-light ratio. In addition, the radial intensity profiles of both samples (local and high redshift Tadpoles) show an exponential decrease at large galactocentric distances, which we interpreted as an evidence for the existence of an underlying disk.

From the point of view of their chemical content, Extremely Metal Poor (XMP) galaxies are the least evolved objects in the local universe (Pagel et al., 1992; 

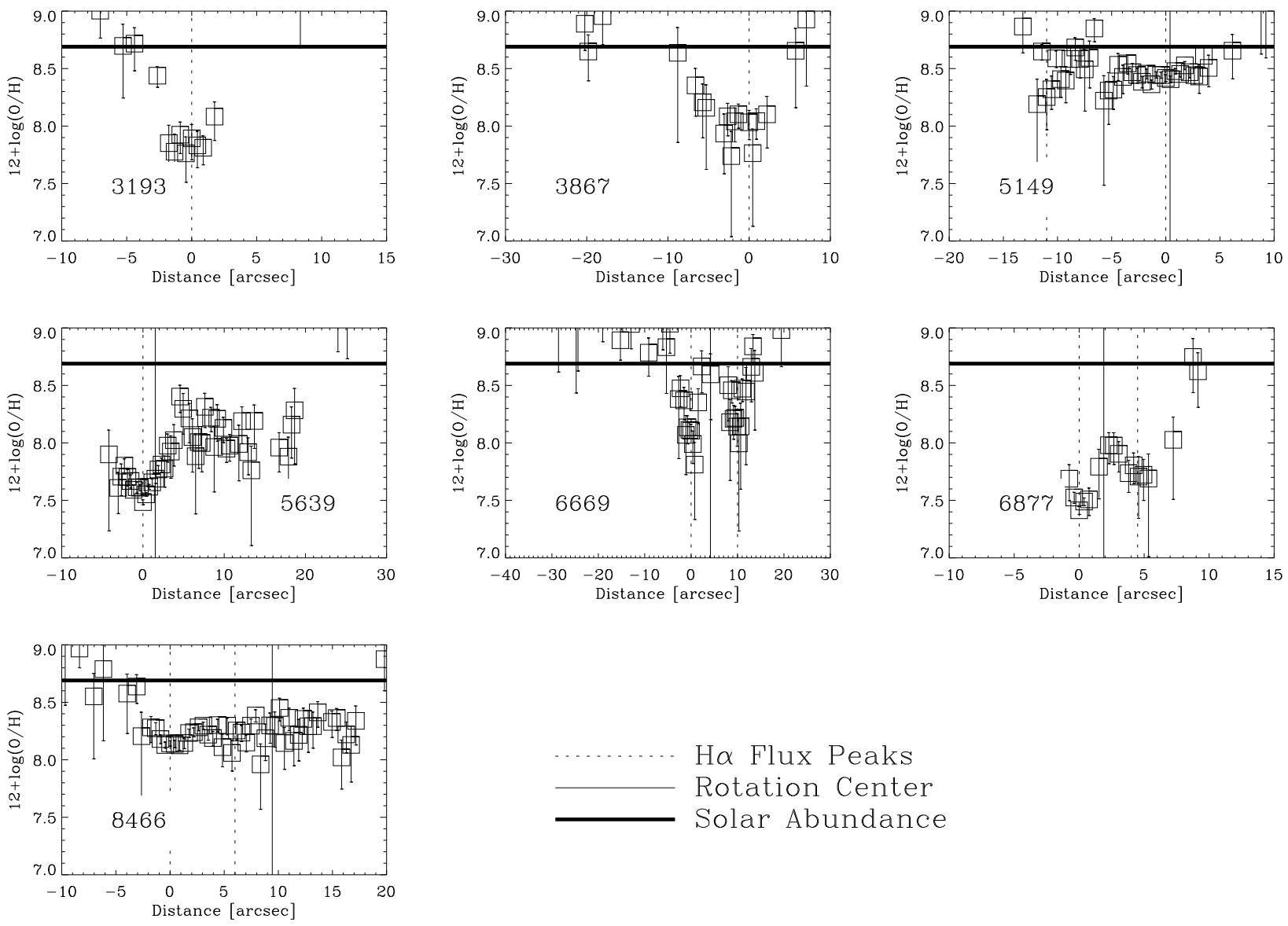

Figure 5: Oxygen abundance variation across the galaxies. The vertical solid line represents the center of rotation, whereas the vertical dotted line indicates the location of the maximum in the $\mathrm{H} \alpha$ flux profile. The thick horizontal solid line indicates the solar metallicity. Note the existence of abundance variations, with the minima coinciding with the largest $\mathrm{H} \alpha$ signals. Note also that KISO5639 and KISO6877 reach very low abundances, below one-tenth of the solar value; therefore, they are members of the set of rare XMP galaxies.

Kunth \& Östlin, 2000). They represent only $0.1 \%$ of the galaxies in an arbitrary nearby volume (MoralesLuis et al., 2011) and a significant fraction of these chemically primitive objects turn out to have tadpole or cometary shape (Brinchmann et al. 2008). This association between low metallicity and tadpole shape suggests that both are attributes of very young systems.

Detailed studies of a significative sample of the local Tadpole class by means of high-resolution long-slit spectroscopy put into evidence two important facts: they do rotate and they show oxygen abundances that vary along the disk. A high percentage of them are XMP galaxies and the metallicity is minimum were starformation is maximum. Moreover, the rotation center does not coincide with the current starburst location (the head).

The oxygen metallicity estimated from [NII] $6583 / \mathrm{H} \alpha$ often shows significant spatial gradients across the galaxies $(\sim 0.5 \mathrm{dex})$, being lowest at the head and increasing in the rest of the galaxy, tail included. A similar result with the highest SFR been the lowest metallicity region has also been found in low metallicity GRB host galaxies (Levesque et al., 2011).

The sense of the resulting metallicity gradient differs from the observation of local disk galaxies, where the gas-phase metallicity increases toward the galaxy centers (Vilchez et al., 1988) or is just constant (Genel et al., 2008). However, the type of variation we measure, with a minimum metallicity at the most intense star-forming region, has been observed in galaxies at redshift around 3 by Cresci et al. (2010), where it is interpreted as evidence for infall of pristine gas triggering star formation.

As a result of all the evidence found, we propose that local Tadpole Galaxies are disks still in the process of being formed in our present-day Universe. Moreover 
their otherwise very low star formation is enhanced and triggered by cool-flow accretion of pristine metal-poor gas. This triggering causes an extremely metal-poor head to shows up on the side of an immature (still forming) rotating disk.

There are a number of parallel actions now being undertaken to go further. We have just finished a study of the HI content of a complete sample of XMP galaxies (Fihlo et al., 2013) and compared the properties of the clumps of different galaxy types at different redshifts (Elmegreen et al., 2013). The recent analysis of a new sample corroborates the metallicity drop in the XMP galaxies using the so call direct method (Sánchez Almeida et al. 2014).

Cosmological simulations predict cold-flow buildup to be the main mode of galaxy formation (Dekel et al., 2009). The incoming gas is expected to form giant clumps that spiral in and merge into a central spheroid. To confirm such a scenario at high redshift is neither easy nor feasible and we think we have discovered nearby cases that may allow for comparisons.

We are making a comprehensive study of the disks and bursts of other BCDs with XMP properties to search for extremely metal poor clumps in 2D spectroscopy. The final aim is to characterize the local XMP Galaxy sample within the cool flow paradigm.

\section{Acknowledgement}

This work has been partly funded by the Spanish Ministry for Science, project AYA 2010-21887-C04-04. Results based on observations at the Observatorio del Roque de los Muchachos (ORM), operated by the IAC at La Palma. Thanks to Franco Giovannelli and Lola Sabau-Graziatti, organizers of this workshop for their kindness and dedication. Thanks to the referee for useful comments.

\section{References}

[1] van den Bergh, S., Abraham, R.G., Ellis, R.S., Tanvir, N.R., Santiago, B.X., \& Glazebrook, K.G. 1996, AJ 112, 359

[2] Emegreen, D.M., Elmegreen, B.G., Rubin, D.S. \& Schaffer, M.A. 2005, ApJ, 631, 85 doi:10.1086/432502

[3] Elmegreen, D. M., Elmegreen, B. G., Ravindranath, S., \& Coe, D. A. 2007: ApJ, 658, 763 doi:10.1086/511667

[4] Elmegreen, B. G. \& Elmegreen, D. M. 2010: ApJ, 722, 1895 doi:10.1088/0004-637X/722/2/1895
[5] Straughn, A. N., Cohen, S. H., Ryan, R. E., et al. 2006, ApJ, 639, 724 doi:10.1086/499576

[6] Windhorst, R. A., Cohen, S. H., Straughn, A. N., et al. 2000, NewAR, 50, 821.

[7] Elmegreen, C., Elmegreen, B., Sánchez Almeida, J., Muñoz-Tuñón, C., Putko, \& Dewberry, 2012, ApJ 750, 95. doi:10.1088/0004-637X/750/2/95

[8] Miyauchi-Isobe, N., Maehara, H., \& Nakajima, K. 2010: Publications of the National Astronomical Observatory of Japan, 13, 9.

[9] Sánchez Almeida, J., Muñoz-Tuñón, C., Elmegreen, D.M, et al., 2013: ApJ, 767, 74. doi:10.1088/0004-637X/767/1/74

[10] Amorin, R., Muñoz-Tuñón, C., Aguerri, J.A., et al., 2007, A\&A, 467, 541.

[11] Amorin, R., Aguerri, J.A., Muñoz-Tuñón, C., et al., 2009, A\&A, 501, 75.

[12] Sánchez Almeida, J., Muñoz-Tuñón, C., Amorin, et al., 2008, ApJ., 685, 194. doi:10.1086/590380

[13] Morales-Luis, A.B., Sánchez Almeida, J., Aguerri, J.A. \& Muñoz-Tuñón: 2011, ApJ, 743, 77. doi:10.1088/0004-637X/743/1/77

[14] Sánchez Almeida, J., Aguerri, J. A. L., MuñozTuñón, C.,\& Vazdekis, A. 2009, ApJ., 698, 1497. doi:10.1088/0004-637X/698/2/1497

[15] Asplund, Grevesse, Sauval \& Scott: 2009 ARA\&A, $47,481$.

[16] Pagel, B., Simonson, E.A., Terlevich, R. \& Edmunds, M., 1992, MNRAS, 255, 325.

[17] Kunth, D. \& Östlin, G. 2000: ARAA, 10, 1.

[18] Papaderos, P., Guseva, N. G., Izotov, Y. I., \& Fricke, K. J. 2008: A\&A, 491, 113.

[19] Brinchmann, J., Kunth, D. \& Durret, F., 2008: $\mathrm{A} \& \mathrm{~A}, 485,657$.

[20] Levesque, E.M., Berger, E., Soderberg, A.M. \& Chornock, R., 2011, ApJ, 739, 23. doi:10.1088/0004-637X/739/1/23

[21] Vilchez, J. M., Pagel, B., Diaz, A. I., Terlevich, E., \& Edmunds, M. G. 1988, MNRAS, 235, 633.

[22] Genel, S., Naab, T., Genzel, R., Förster Schreiber, Sternberg, Oser, Johansson, Davé, Oppenheimer, \& Burkert, 2012: ApJ, 745, 11 doi:10.1088/0004-637X/745/1/11

[23] Cresci, G., Mannucci, F., Maiolino, R. et al., 2010, Natur, 467, 811. doi:10.1038/nature09451 
[24] Filho, M. E. , Winkel, B., Sánchez Almeida et al., [25] Dekel, A., Birnboim, Y., Engel, G., Freundlich, 2013, A\&A, 558, 18. Goerdt, Mumcuoglu, Neistein, Pichon, Teyssier, \& Zinger, 2009: Nature 457, 451.

[Elmegreen et al., 2013] , 2013, ApJ., 774, 86. [26] Sánchez Almeida, J., Morales-Luis, A.B, Muñozdoi:10.1038/nature07648 Tuñón, C., et al., 2014: ApJ in press. 